\title{
Ternary critical point determination of experimental demixion curve: calculation method, relevance and limits
}

\author{
C. Goutaudier ${ }^{1}$, F. Bonnet ${ }^{2}$, R. Tenu ${ }^{1}$, O. Baudouin ${ }^{2}$, and J.J. Counioux ${ }^{1}$ \\ ${ }^{1}$ Laboratoire des Multimatériaux et Interfaces, UMR 5615 Université Lyon 1/CNRS, 69622 Villeurbanne, France \\ ${ }^{2}$ Prosim SA, Immeuble Stratège A, 51 rue Ampère, F-31670 Labège, France
}

\begin{abstract}
In many cases of miscibility gap in ternary systems, one critical point at least, stable or metastable, can be observed under isobaric and isothermal conditions. The experimental determination of this invariant point is difficult but its knowledge is essential. The authors propose a method for calculating the composition of the invariant solution starting from the composition of the liquid phases in equilibrium. The computing method is based on the barycentric properties of the conjugate solutions (binodal points) and an extension of the straight diameter method. A systematic study was carried out on a large number of ternary systems involving diverse constituents (230 sets ternary systems at various temperatures). Thus the results are presented and analyzed by means of consistency tests.
\end{abstract}

\section{Introduction}

According to the Gibbs' phase rule, the knowledge of all the invariant transformations permits to predict qualitatively each of the equilibrium states of a multiphase system. Hence, the importance of their determination implies the development of many methods and techniques applied to their characterization. However, among invariant equilibria, the critical phenomena, which we find particularly by studying the miscibility gap in a multicomponent system, occupy a special place. This problem can be illustrated by a demixing zone in the polythermal diagram or, more simply, in an isotherm of a ternary system (figure 1). The binodal curves and surfaces are continuous in a mathematical sense but, thermodynamically separated by a critical point or line. This isobaric and isothermal invariant point $\left(\kappa_{\mathrm{T}}\right)$ or monovariant line $\left(\kappa-\kappa_{\mathrm{T}}\right)$ is difficult to reach by the direct measurement. So, a calculation method was developed and tested on numerous isothermal ternary systems with large demixing zone. The semi-empirical method consists in using the experimental results for the different tie-lines. A database was constructed from experimental data taken from the National Institute of Standards Technology NIST [1].

The determination of each critical point was conducted in two steps: the first one is based on a generalization of the straight diameter method and the second, on the exploitation of the modulus of the experimental tie-lines.

This contribution presents the original method of calculation derived from the rectilinear diameter law and the analysis of results shows its limits and its relevance.

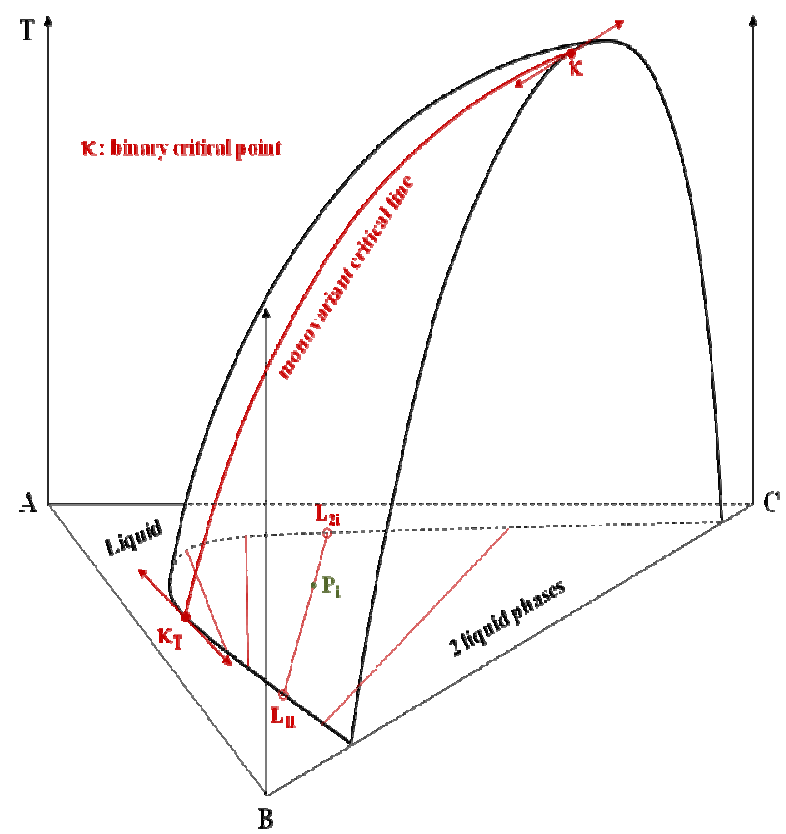

Figure 1. Shell defining the domain of liq-liq equilibrium in the $3 \mathrm{D}$ representation of the ternary system $\mathrm{A}+\mathrm{B}+\mathrm{C}$ under constant pressure $\left(\kappa_{\mathrm{T}}\right.$ : ternary invariant critical point).

\section{Methodology}

\subsection{Database}

A database was built, containing more than 90 ternary systems and around 230 sets of experimental data at various temperatures.

Experimental data have been extracted from NIST database [1]. The selected systems have a wide miscibility gap with a sufficient number of experimental 
points. The required data are the compositions of the two conjugated liquid phases, the temperature and if they are available the coordinates of the invariant point.

The database is organized in order to use easily the data to test the method in order to assess its reliability.

\subsection{Straight diameter method}

Originally, the formulation of the so-called law of rectilinear diameter for the determination of the critical volume of substances became in a very important tool for researchers in the field of critical phenomena [2]. This semi-empirical method consists in plotting the midpoints of each tie-line, defining the associated straight line with the better regression parameters, and then extrapolating this latter until its intersection with the experimental binodal curve [3]. However, this graphical method needs a fairly good symmetry between both sides of the binodal curve, which provides a good linearity of the midpoints. In order to apply this property to unsymmetrical curves, we have performed a barycentric balance of the two conjugate points. For each tie-line (i), the barycentric point $\mathrm{P}_{\mathrm{i}}$ satisfies the following relation:

$\overrightarrow{\mathrm{P}_{\mathrm{i}}}=(1-\lambda) \overrightarrow{\mathrm{L}_{1 \mathrm{i}}}+\lambda \overrightarrow{\mathrm{L}_{2 \mathrm{i}}}$

where (1- $\lambda)$ and $\lambda$ represent respectively the mass fractions of the liquids $\mathrm{L}_{1 \mathrm{i}}$ and $\mathrm{L}_{2 \mathrm{i}}$ in equilibrium at the study temperature. In the pedagogic system $\mathrm{A}+\mathrm{B}+\mathrm{C}$ (figure 1), the components of the column vectors $\overrightarrow{\mathrm{P}_{\mathrm{i}}}$, $\overrightarrow{\mathrm{L}_{1 \mathrm{i}}}$ and $\overrightarrow{\mathrm{L}_{2 \mathrm{i}}}$ are their mass fractions in the solvent $\mathrm{A}$, the constituent $\mathrm{B}$ (x-axis) and the constituent $\mathrm{C}$ (y-axis).

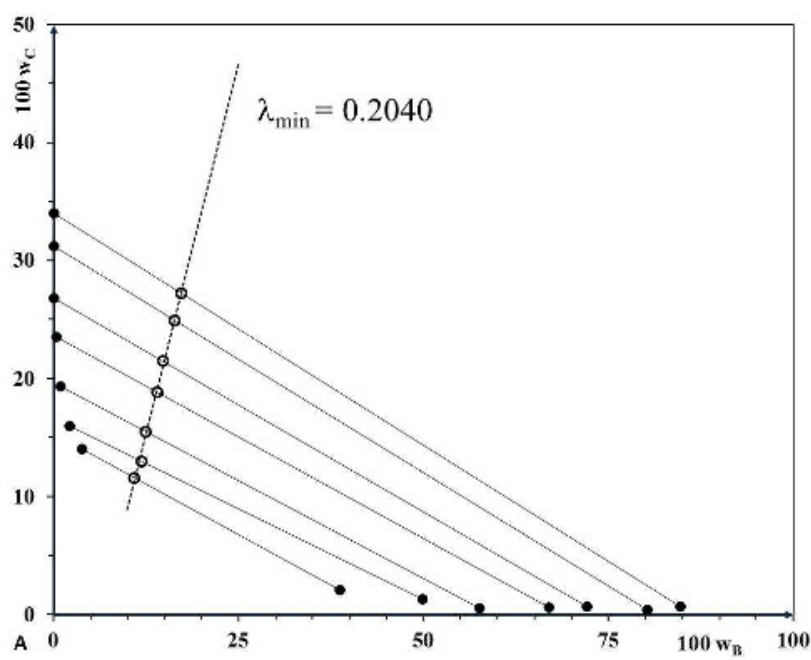

Figure 2. Graphical representation of the straight diameter method in the ternary system $\mathrm{A}+\mathrm{B}+\mathrm{C}$ (isotherm and isobar).

The straight line $\mathrm{D}_{\lambda}$ corresponds to the better regression parameters for $\lambda_{\text {min }}$.

For a series of tie-lines of the same isotherm, an error function $\mathrm{E}$ is then calculated as function of the parameter $\lambda$, which optimal value $\lambda_{\min }$ corresponds to the minimum of $E$ ie the best fitting parameters. The equation of the corresponding straight line $\mathrm{D}_{\lambda}$ may be written (figure 2):
$\mathrm{WC}=\mathrm{aWB}+\mathrm{b}$

where $\mathrm{W}_{\mathrm{B}}$ and $\mathrm{W}_{\mathrm{C}}$ are respectively the mass fractions of the constituents $\mathrm{B}$ and $\mathrm{C}$. Obviously $\mathrm{a}$ and $\mathrm{b}$ are the calculated parameters of the straight line $\mathrm{D}_{\lambda}$. According to the rectilinear diameter law, this line will cut the binodal curve near the critical point.

\subsection{Modulus method}

The second step of calculation is based on a particular property of the critical points: at this point indeed, the internodal length becomes zero. Since each tie-line can be identified by its intersection with the straight line $D_{\lambda}$ previously obtained by the diameter method, the length of the tie-line can be considered as a function of the abscissa or the ordinate of this point. As the variation law is unknown, it was assumed that the raising of the internodal distance to the $\alpha^{\text {th }}$ power $\left(\mathrm{L}^{\alpha}\right)$ linearly varies with the mass fractions $\mathrm{W}_{\mathrm{B}}$ or $\mathrm{W}_{\mathrm{C}}$ of the constituents $\mathrm{B}$ and $\mathrm{C}$. At each tie-line (i), a couple of values $\left(\mathrm{W}_{\mathrm{Bi}}, \mathrm{W}_{\mathrm{Ci}}\right)$ can be calculated by using the equation (2).

For the series of experimental tie-lines, an error function $\mathrm{E}$ is then calculated as function of the parameter $\alpha$, which optimal value $\alpha_{\text {min }}$ corresponds to the common minimum of the $\mathrm{E}$ function, with $\mathrm{W}_{\mathrm{B}}$ or $\mathrm{W}_{\mathrm{C}}$ ordinates. In the figure 3 , we have plotted the modulus $L$ of this length at the power $\alpha_{\min }$ as a function of $\mathrm{W}_{\mathrm{B}}$ or $\mathrm{W}_{\mathrm{C}}$. On a very short distance, we have then extrapolated the straight lines obtained up to the modulus zero. So this extrapolation leads to the coordinates of the critical point $\kappa_{\mathrm{T}}$.

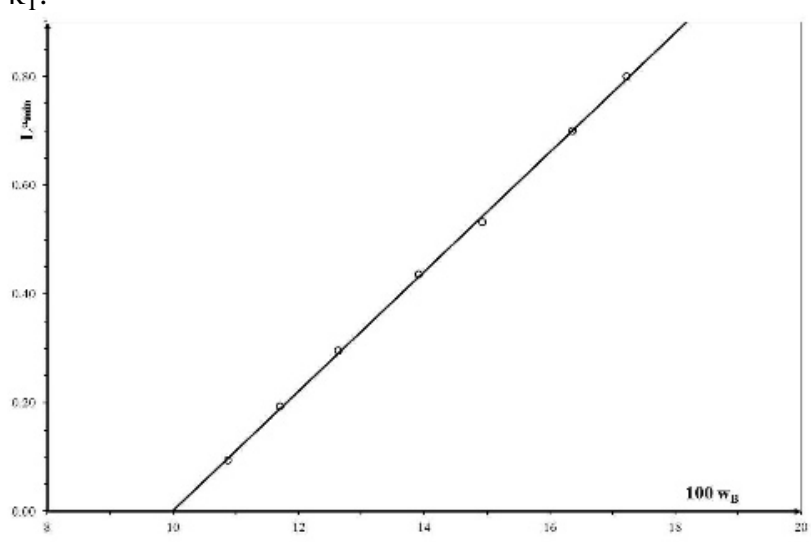

Figure 3. Power $\alpha$ of the tie-line's internodal distance $\mathrm{L}$ as a function of the mass percentage of component B. Extrapolation to zero length corresponds to one of the coordinates of the critical point $\kappa$.

\section{Results}

The proposed method for the determination of the critical point has been tested on 230 experimental ternary systems having a liquid-liquid equilibrium area. It gives consistent results in most cases, provided to have a sufficient number of tie-lines.

Two error functions have been tested in order to find the most suitable regression parameters for each step of the calculation $(\lambda$ and $\alpha)$. The first one was the classical 
correlation coefficient $\mathrm{R}$. The second one was defined as follow:

$$
\begin{aligned}
& \mathrm{E}=\frac{1}{\mathrm{~N}} \sum_{\mathrm{i}}\left(\mathbf{L}_{\mathrm{i}}^{\alpha}-\mathbf{L}_{\mathrm{calc}}^{\alpha}\right)\left(\mathrm{W}_{\mathrm{Bi}}-\mathrm{W}_{\mathrm{Bcalc}}\right)_{\text {or }} \\
& \frac{1}{\mathrm{~N}} \sum_{\mathrm{i}}\left(\mathrm{L}_{\mathrm{i}}^{\alpha}-\mathrm{L}_{\mathrm{calc}}^{\alpha}\right)\left(\mathrm{W}_{\mathrm{Ci}}-\mathrm{W}_{\mathrm{Ccalc}}\right)
\end{aligned}
$$

where $\mathrm{N}$ is the number of experimental tie-lines.

As an example, figure 4 compares the changes of the error functions calculated from the system 2Propanol+Toluene+Water. The 13 experimental tie lines were determined by Washburn at $298 \mathrm{~K}$ under atmospheric pressure using refractive indices measurements [4]. In this figure the variations are plotted in normalized arbitrary unit and lead to the quite similar minimum value.

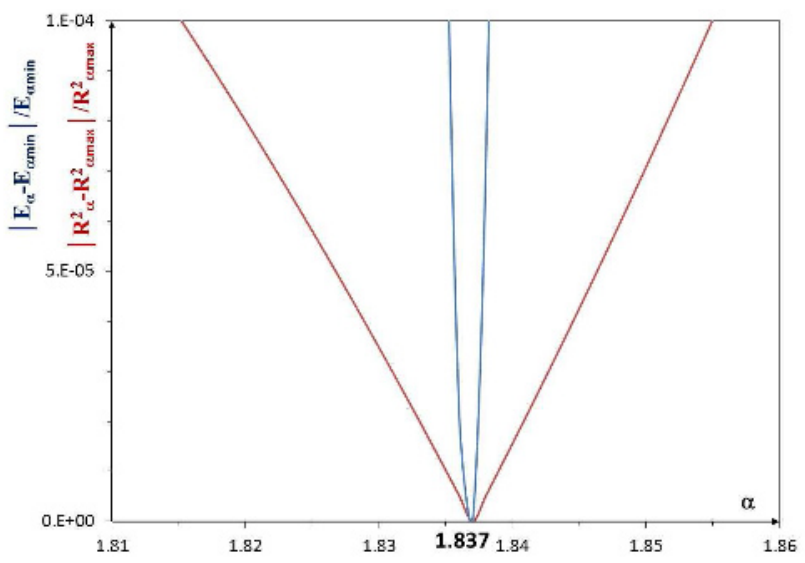

Figure 4. Comparison of the changes of the error functions calculated from the system 2-Propanol+Toluene+Water. ( $\mathrm{R}$ and $E$ values are plotted in normalized arbitrary unit. Experimental data from [4]).

In these conditions, the optimal fitting parameters for the studied case are $\lambda=0.527$ and $\alpha=1.837$.

Then in order to assess the reliability of the calculation method, the coordinates of the critical point are compared with those available in the literature or calculated by classical method using binary interaction parameters. Typical values are given in table 1 for the system 2-Propanol + Toluene + Water at 298K and are in a very good agreement.

Table 1. Coordinates of the critical point $\kappa_{\mathrm{T}}$ calculated from several methods (in molar fraction; $\mathrm{T}=298 \mathrm{~K}$ ).

\begin{tabular}{|c|c|c|c|}
\hline Method & $\begin{array}{c}2- \\
\text { Propanol }\end{array}$ & Toluene & Water \\
\hline NRTL & 0.29 & 0.06 & 0.65 \\
\hline UNIQUAC & 0.30 & 0.075 & 0.625 \\
\hline This work & 0.289 & 0.058 & 0.653 \\
\hline
\end{tabular}

In contrast, the method does not work in a number of cases. Often critical analysis of the experimental results provides the solution easily, as shown in figure 5 for the ternary system Benzene + Hexane + Perfluorohexane. It should check the experimental tie-lines in order to remove aberrant lines intersecting.

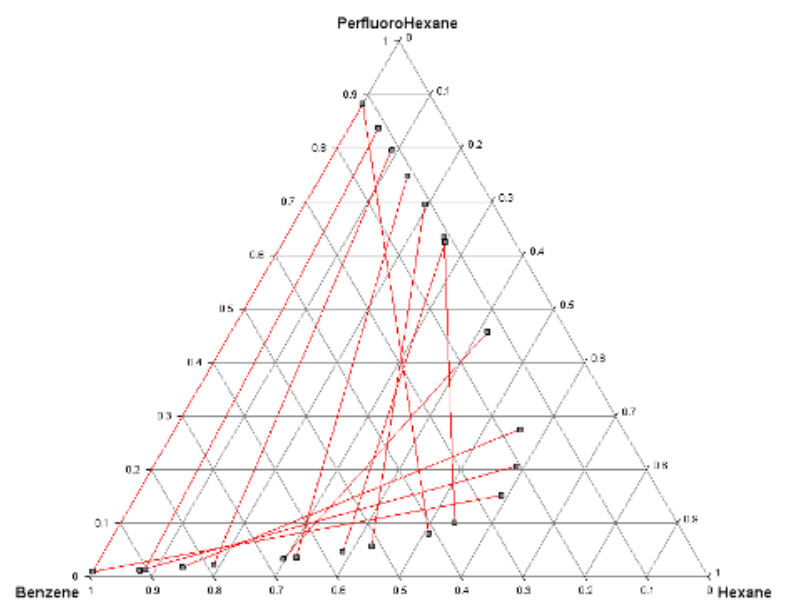

Figure 5. An example of ternary system for which the method of rectilinear diameter cannot work [1].

\section{Conclusion}

The knowledge of the ternary critical point of demixion areas is essential for any definition of an operation unit based on liquid-liquid extraction. The original calculation method was assessed on a large number of experimental data. The method derives from the rectilinear diameter law on which a barycentric balance is applied. The quality of the conjugated experimental points is the dominant parameter.

\section{References}

1. M. Frenkel, R.D. Chirico, V. Diky, C.D. Muzny, A.F. Kazakov, J.W. Magee, I.M. Abdulagatov, K. Kroenlein, C.A. Diaz-Tovar, J.W. Kang, R. Gani, NIST ThermoData Engine Version 7.0, Database \#103B, NIST Thermodynamics Research Center (2011)

2. L.P. Cailletet, E. Mathias, C. R. Séances Acad. Sci. 1021002 (1886); J. Phys. Théor. Appl. 5549 (1886)

3. S. Reif-Acherman, Quim. Nova. 332003 (2010)

4. E.R. Washburn, A.E. Beguin, J. Am. Chem. Soc. 62 $579(1940)$ 